ARTICLE

Received 15 Aug 2014 | Accepted 20 Oct 2014 | Published 26 Nov 2014

DOl: $10.1038 /$ ncomms 6603

OPEN

\title{
Community-integrated omics links dominance of a microbial generalist to fine-tuned resource usage
}

Emilie E. L. Muller ${ }^{1, \star}$, Nicolás Pinel ${ }^{1,2, \star}$, Cédric C. Laczny ${ }^{1}$, Michael R. Hoopmann ${ }^{2}$, Shaman Narayanasamy ${ }^{1}$, Laura A. Lebrun ${ }^{1}$, Hugo Roume ${ }^{1, \dagger}$, Jake Lin ${ }^{1}$, Patrick May ${ }^{1}$, Nathan D. Hicks ${ }^{3}$, Anna Heintz-Buschart ${ }^{1}$, Linda Wampach ${ }^{1}$, Cindy M. Liu ${ }^{3}$, Lance B. Price ${ }^{3}$, John D. Gillece ${ }^{3}$, Cédric Guignard ${ }^{4}$, James M. Schupp ${ }^{3}$, Nikos Vlassis ${ }^{1, \dagger}$, Nitin S. Baliga ${ }^{2}$, Robert L. Moritz ${ }^{2}$, Paul S. Keim ${ }^{3} \&$ Paul Wilmes $^{1}$

Microbial communities are complex and dynamic systems that are primarily structured according to their members' ecological niches. To investigate how niche breadth (generalist versus specialist lifestyle strategies) relates to ecological success, we develop and apply an integrative workflow for the multi-omic analysis of oleaginous mixed microbial communities from a biological wastewater treatment plant. Time- and space-resolved coupled metabolomic and taxonomic analyses demonstrate that the community-wide lipid accumulation phenotype is associated with the dominance of the generalist bacterium Candidatus Microthrix spp. By integrating population-level genomic reconstructions (reflecting fundamental niches) with transcriptomic and proteomic data (realised niches), we identify finely tuned gene expression governing resource usage by Candidatus Microthrix parvicella over time. Moreover, our results indicate that the fluctuating environmental conditions constrain the accumulation of genetic variation in Candidatus Microthrix parvicella likely due to fitness trade-offs. Based on our observations, niche breadth has to be considered as an important factor for understanding the evolutionary processes governing (microbial) population sizes and structures in situ.

\footnotetext{
${ }^{1}$ Luxembourg Centre for Systems Biomedicine, University of Luxembourg, 7 Avenue des Hauts-Fourneaux, L-4362 Esch-sur-Alzette, Luxembourg. ${ }^{2}$ Institute for Systems Biology, 401 Terry Avenue North, Seattle, Washington 98109, USA. ${ }^{3}$ TGen North, 3051 West Shamrell Boulevard, Flagstaff, Arizona 86001, USA. ${ }^{4}$ Centre de Recherche Public-Gabriel Lippmann, 41 rue du Brill, L-4422 Belvaux, Luxembourg. ${ }^{\star}$ These authors contributed equally to this work. $\dagger$ Present address: Laboratory of Microbial Ecology and Technology, Ghent University, Coupure Links 653, B-9000 Ghent, Belgium (H.R.) or Adobe Research, 345 Park Avenue, San Jose, California 95110, USA (N.V.). Correspondence and requests for materials should be addressed to P.W. (email: paul.wilmes@uni.lu).
} 
$\mathrm{M}$ icroorganisms are ubiquitous and form complex, heterogeneous and dynamic assemblages ${ }^{1}$. They represent essential components of the Earth's biogeochemical cycles ${ }^{2}$, human metabolism ${ }^{3}$ and biotechnological processes ${ }^{4}$. Microbial population sizes and structures are governed by resource availability and usage ${ }^{5-7}$, and mainly develop in response to the breadths of the fundamental and realized niches of constituent populations ${ }^{8,9}$ (narrow for specialists; wide for generalists) albeit being influenced by stochastic neutral processes ${ }^{8,9}$. Microbial niche breadths remain poorly described in situ (for an earlier study, see ref. 10). The application of high-fidelity, high-resolution and high-throughput molecular analyses to microbial consortia holds great promise for resolving population-level phenotypes and defining their corresponding niche breadths in situ ${ }^{11}$. However, to obtain community-wide multi-omic data that can be meaningfully integrated and analysed, systematic measurements are essential ${ }^{1}$.

We have recently developed the required laboratory methods that enable us to isolate representative biomolecular fractions (DNA, RNA, proteins and small molecules) from single microbial community samples ${ }^{12,13}$. Here, we expand this concept by performing integrated omic analyses of purified biomolecular fractions from oleaginous mixed microbial communities (OMMCs) located on the surface of an anoxic biological wastewater treatment tank to study how microbial lifestyle strategies relate to ecological success and the associated community-level phenotypes in this fluctuating but wellcharacterized environment. In addition, OMMCs are typically enriched in lipid-accumulating filamentous bacteria and often associated with operational difficulties, such as phase separation and bulking in biological wastewater treatment plants ${ }^{14}$. However, the phenotypic traits of OMMCs may allow for the recovery of lipids from wastewater streams for subsequent chemical energy recovery through biodiesel synthesis ${ }^{15}$. As for other microbial consortia, a detailed understanding of OMMC ecology is essential for the formulation of strategies to shape microbial community structure and function (in this case, enriching for lipid-accumulating bacteria) in the future. Building on the recently developed methodologies for the systematic molecular characterization of microbial consortia ${ }^{12,13}$, and for resolving and reconstructing populationlevel genomic complements from community-wide sequence data ${ }^{16}$, here, we integrate multi-omic data sets to resolve microbial lifestyle strategies in situ, identify finely tuned gene expression governing resource usage by a dominant bacterial generalist population and reveal that genetic variation within this population is constrained likely due to fitness trade-offs.

\section{Results}

Coupled metabolomic-taxonomic analyses over space and time. To obtain a detailed view of OMMC (Supplementary Fig. 1a) lipid accumulation and bacterial composition, we first applied coupled metabolomics and 16S rRNA gene sequencing to samples taken over space and time (see the Methods section). The initial sample set included four distinct biological replicates (Supplementary Fig. 1b) from four representative time points (two in autumn, two in winter; Methods and Supplementary Fig. 2a,b). Using gas chromatography coupled to tandem mass spectrometry (GC-MS/MS), absolute quantifications of the 14 major long-chain fatty acids were obtained for OMMC biomass and wastewater, respectively (Methods). The V3 and V6 hypervariable regions of the $16 \mathrm{~S}$ rRNA gene were amplified from the DNA fraction of the samples (Methods). The barcoded amplicons were pyrosequenced on a 454 GS FLX platform, yielding a total of 265,592 reads $(n=10,574 \pm 3,451$ (mean \pm s.d.) per OMMC sample after quality control and chimera filtering). Direct taxonomic classification of the obtained sequencing reads demonstrates that, at the phylum level, the OMMCs of the studied treatment plant were dominated across the studied seasons by Proteobacteria and Actinobacteria, which constituted $43 \% \pm 14 \%$ and $21 \% \pm 5 \%$ (mean \pm s.d.) of the community, respectively (Fig. 1a). Similar results were obtained when operational taxonomic unit clustering was applied before classification (Supplementary Fig. 3a). Among the two most dominating taxa over time (Fig. 1b; Supplementary Fig. 3b), Candidatus Microthrix spp., a well-known lipid-accumulating genus ${ }^{17,18}$, correlated with a more pronounced community-wide lipid accumulation phenotype (Spearman correlation coefficient $\rho \geq 0.8$ for Candidatus Microthrix spp. and palmitoleic and oleic acids, respectively; Fig. 1c). This trend, despite the metabolic versatility
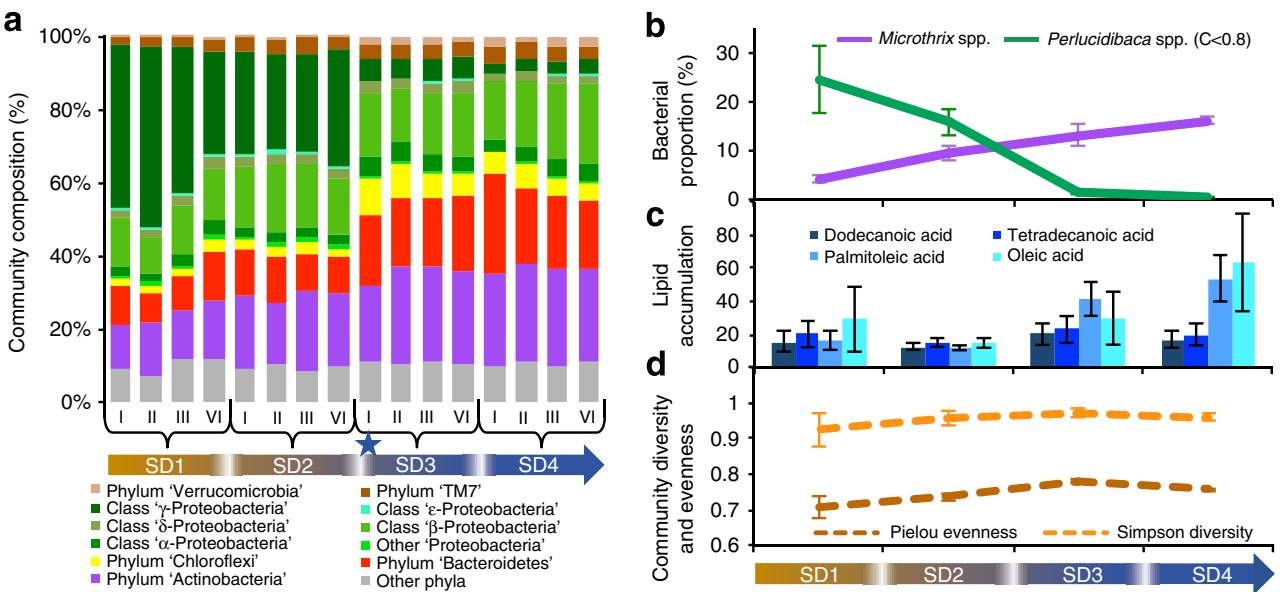

Figure 1 | Microbial community dynamics and lipid accumulation from wastewater. (a) Fractions of taxa identified across the communities sampled on four distinct dates (SD1-SD4). Roman numerals refer to the four biological replicates sampled per time point. The blue star indicates the representative sample from SD3 subjected to the integrated omic analysis. (b) Average genus-level abundances of the two dominant populations. The most abundant microbial population in winter was identified as Candidatus Microthrix spp., whereas a population tentatively identified (confidence level $<0.8$ ) as Perlucidibaca spp. was dominant in autumn. (c) Long-chain fatty acid intracellular accumulation per sampling date expressed as ratios between quantified intracellular and extracellular long-chain fatty acid abundances. (d) Genus-level alpha diversity and evenness. (b-d), error bars represent s.d. $(n=4)$. 
of Candidatus Microthrix spp. ${ }^{17,18}$ and the statistically significant lower levels of lipids available compared with other carbon and energy sources particularly in winter (Wilcoxon rank-sum test, $P<0.001, n=15$; Supplementary Fig. 4), suggests optimal foraging behaviour ${ }^{19,20}$ by Candidatus Microthrix spp.

High-throughput multi-omic analyses. To obtain an initial view of the population-level characteristics that determine the ecological success of Candidatus Microthrix spp. in winter in comparison with other co-occurring microbial populations, we first conducted a detailed integrated omic analysis of a single representative sample obtained on sampling date 3 (SD3; Methods). This sample was selected on the basis of the relatively large Candidatus Microthrix spp. population size (Fig. 1b), the desired community-wide lipid accumulation phenotype (Fig. 1c) and its even but diverse community composition (Fig. 1d). Concomitantly isolated DNA, RNA and protein fractions were processed and subjected to high-throughput metagenomic, metatranscriptomic and metaproteomic analyses. Massive parallel sequencing of DNA and $\mathrm{CDNA}$ resulted in the generation of $1.47 \times 10^{7}$ and $1.65 \times 10^{7}$ metagenomic and metatranscriptomic paired-end reads, respectively. Shotgun proteomic analyses based on liquid chromatography followed by tandem mass spectrometry (LC-MS/MS) resulted in the generation of 271,915 mass spectra (Methods).

Assembly-free community profiling. To assess the composition of the winter OMMC, we first carried out an assembly-free community analysis. For this, the results obtained using two shotgun sequence data profiling tools, namely MetaPhlAn ${ }^{21}$ and MG-RAST ${ }^{22}$, were compared with the profiles obtained using the previous $16 \mathrm{~S}$ rRNA gene sequencing. Given the poor taxonomic classification of Candidatus Microthrix spp. in the databases used by these profiling tools, all analyses were limited to phylum-level classification. At this level, similar community structures were apparent for the representative sample from SD3 using the three distinct approaches (Supplementary Fig. 3a and Methods).

Second, to resolve the functions encoded and expressed by the OMMC from SD3, the proportions of genes and transcripts belonging to different cluster of orthologous group (COG) functional categories were compared for both the metagenomic and metatranscriptomic data sets (Supplementary Fig. 5). Similar proportions were observed for most of the different functional categories in both data sets. Nevertheless, major differences were observed for the categories 'J-translation, ribosomal structure and biogenesis', ' $\mathrm{O}-$ posttranslational modification, protein turnover, chaperones', and ' $\mathrm{C}$-energy production and conversion'. Differences in gene copy numbers and transcript abundances may be expected for these functional genes because of their typical high levels of constitutive expression. The proportion of gene copies and transcript numbers were similar for the COG category 'I-lipid transport and metabolism' although these genes are expected to have essential roles in OMMCs and, therefore, overall high levels of expression may be expected. The previous findings suggest that key members in the OMMC, that is, Candidatus Microthrix spp., are involved in lipid transformations. Consequently, key processes related to lipid transport and metabolism, that is, resource usage, have to be resolved at the population level. Therefore, to deconvolute the activities of the constituent OMMC members, a detailed population-resolved analysis was subsequently performed.

Population-resolved integrated omic analyses. To resolve the traits of the dominant populations within the community obtained on SD3, composite genomes (CGs) were reconstructed using a newly developed iterative binning and de novo assembly procedure for the combined metagenomic and metatranscriptomic sequence data (Methods). Detailed profiling and grouping of the assembled contiguous sequence fragments (contigs) were performed on the basis of centred log-ratio transformed pentanucleotide signatures and visualization using the Barnes-Hut Stochastic Neighbour Embedding (BH-SNE) algorithm ${ }^{16}$, followed by human-augmented clustering (Methods). Using this approach, we identified nine CG groups (Fig. 2a) that displayed homogeneous $\mathrm{G}+\mathrm{C}$ percentage (Fig. 2b). The assembled contigs from the nine CG groups were subjected to gene calling and annotation (Methods), which led to the identification of 23,317 coding sequences, with 16,841 and 1,533 of these being represented at the transcript and protein levels, respectively.

The refinement of the CGs by depth of read coverage resulted in the splitting of CG8 into low (CG8a) and high (CG8b) coverage populations (Supplementary Fig. 6). The average amino-
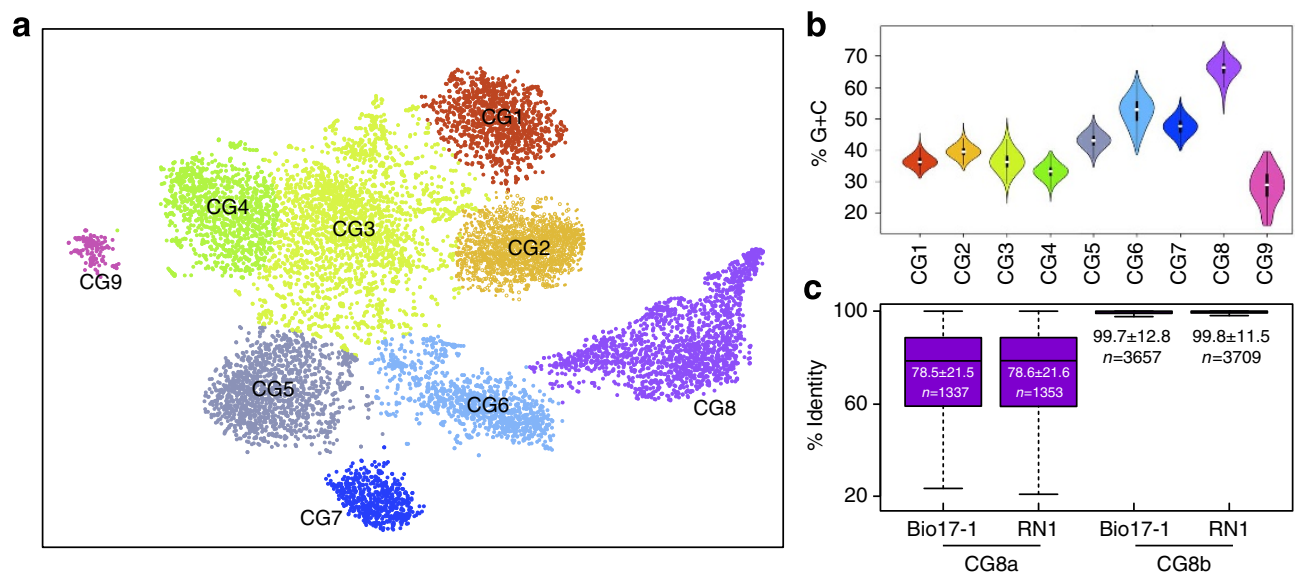

Figure 2 | Identification of genomic fragments derived from distinct populations. (a) Binning of assembled contigs ( $\geq 1,000$ bp in length) on the basis of pentanucleotide signatures and visualization using the BH-SNE algorithm followed by human-augmented clustering of composite genome (CG) groups. (b) Violin plots of the $\mathrm{G}+\mathrm{C}$ percentage for contigs within each of the CG groups. (c) Percentage amino-acid identity of the two subpopulations in CG8 (CG8a and CG8b) compared with the two sequenced Candidatus Microthrix parvicella (Bio17-1 (ref. 16) and RN1 (ref. 17)) genomes. The values are median \pm s.d. and $n$ is the number of putative orthologues identified as best BLAST hits. Boxplots represent the lower quartile, median and upper quartile. Whiskers are placed at $\times 1.5$ interquartile range beyond the lower and upper quartiles. 
acid sequence identities of CG8b with recently obtained genome sequences of Candidatus Microthrix parvicella strains Bio17-1 (ref. 17) and RN1 (ref. 18) were $>99 \%$ (Fig. 2c), an identity level usually observed among strains of the same bacterial species ${ }^{23}$. In contrast, the CG8a identity levels compared with the same reference sequences were around $78 \%$ (Fig. 2c). Consequently, CG8b represents a Candidatus Microthrix parvicella population in this OMMC sample from SD3.

The identities of the other CGs were determined using 31 phylogenetic marker genes ${ }^{24}$ resulting, for example, in the tentative identification of CG5 as a population belonging to the Moraxellaceae family of the $\gamma$-Proteobacteria (Supplementary Data 1).

Eight of the 10 reconstructed CGs were estimated to be $>60 \%$ complete with CG8b, CG4 and CG5 being 97.5, 90 and $85 \%$ complete, respectively (Supplementary Data 1). On the basis of population sizes inferred from the mapping of the metagenomic read data onto the CGs (Methods), these three community members represent the first, the seventh and the fourth most abundant OMMC populations, respectively. Because CG8b and CG5 represent the most deeply covered nearly complete genomic reconstructions, a detailed analysis of the ecophysiology of these populations based on the generated functional omic data was performed.

By mapping the metatranscriptomic and metaproteomic data onto the reconstructed CGs (Methods), all 10 populations were found to be active albeit exhibiting differing levels of gene expression (Fig. 3a, Supplementary Figs 7-9, Supplementary Data 2). Observed patterns of gene expression were not necessarily consistent at the transcript and protein levels for the different CGs. Discrepancies between the levels of gene expression inferred from transcriptomic and proteomic data have been well described in eukaryotes ${ }^{25}$ and these have also recently been observed for microbial communities ${ }^{26}$. The lack of correlation may have different reasons including differing molecular halflives $^{26}$ and/or possible posttranscriptional or posttranslational modifications, which are not detectable using the transcriptomic and proteomic methodologies used in this study.

Despite its large population size, population CG8b expressed only a comparatively small fraction $(45.8 \%$ of possible transcripts detected) of its genetic complement in situ (Fig. 3b, Supplementary Data 1, Supplementary Fig. 8) and this at a moderate level of expression both at the transcript and protein levels (Supplementary Fig. 9a), suggesting the fine-tuning of gene expression by CG8b. On the contrary, the other CGs exhibited expression of the majority of their genetic repertoire (Fig. 3, Supplementary Data 1, Supplementary Fig. 8). In particular, $92.7 \%$ of CG5 genes were detected at the transcript level.

On the basis of its genetic repertoire, Candidatus Microthrix parvicella appears to be physiologically versatile ${ }^{17,18}$ which, combined with its enrichment under fluctuating environmental conditions, indicates a generalist lifestyle strategy ${ }^{27}$. The finetuning of gene expression is particularly apparent for genes involved in lipid transport and metabolism, which show a clear genomic enrichment within the CG8b population although only a limited subset, that is, $46 \%$, are expressed (Fig. 3b, Supplementary Fig. 8). Among these genes, long-chain fatty acid-CoA ligases are essential for the assimilation and activation of extracellular fatty acids into their acyl-CoA conjugates ${ }^{28}$ and are therefore required for resource usage by Candidatus Microthrix parvicella. CG8b encodes 29 genes annotated as homologues of this enzyme class, indicating that a broad spectrum of free fatty acids may be assimilated by this population. Only 11 and 14 of these genes were found to be expressed at the RNA and protein levels, respectively (Fig. 4a). In contrast, the five genes annotated as long-chain fatty acid-CoA ligase homologues in CG5 were all expressed (Supplementary Data 2). This observation suggests the fine-tuned expression of these genes by Candidatus Microthrix parvicella, likely through the tight regulation of gene expression, to facilitate preferential resource usage in accordance with optimal foraging behaviour ${ }^{19,20}$.

Population-level genetic diversity. To assess the overall frequencies of population-level genetic variation and determine how these variations may reflect the lifestyle strategy of CG8b, the number of single-nucleotide polymorphisms (SNPs) identified in individual CGs were normalized according to reconstructed CG length and population sizes inferred from the proportion of metagenomic reads mapped to the reconstructed CGs (Supplementary Data 1). CG8b displayed a relatively limited level of genetic variation. For example, it exhibited one order of magnitude fewer SNPs compared with CG5, the other almost complete reconstructed CG with enough coverage to confidently infer SNP densities (Supplementary Data 1). Given the generalist lifestyle strategy of CG8b, the relatively high within-population genetic homogeneity may be explained by fitness trade-offs resulting, for example, from antagonistic pleiotropy ${ }^{29,30}$. In a fluctuating environment, most of the beneficial or neutral mutations under one condition may be deleterious under other conditions, thereby restricting the evolutionary rate of generalists $^{29,30}$. An alternative hypothesis positing that this low population-level variation may be due to a recent genetic bottleneck (selective sweep, colonization, and so on.) followed by population expansion ${ }^{31}$ may be rejected on the basis of the high genetic similarity between the reconstructed CG and the two available Candidatus Microthrix parvicella genome sequences from strains isolated from distant wastewater treatment plants 7 and 16 years before the present study (Fig. 2c).

Fine-tuned gene expression and limited genetic diversity over time. To validate the previous snapshot views of the ecophysiology and structure of populations CG8b and CG5, identical multi-omic analyses were carried out on three additional, rationally selected OMMC samples from the same wastewater treatment tank (Methods). A first additional sample was collected on SD7 approximately a year after SD3 when the measured physico-chemical parameters were very similar to those measured on SD3 (Supplementary Fig. 2b,c). In addition, samples were selected from SD5 and SD6 because the physico-chemical parameters on these dates were at variance with SD3 and SD7 (Supplementary Fig. 2c). Importantly, the additional samples also contain populations CG8b and CG5 at sufficient quantities to obtain the necessary coverages at the genomic and transcriptomic levels for the subsequent analyses of genetic diversity and gene expression over time (Table 1). Massive parallel sequencing of DNA and cDNA resulted in the generation of an additional $5 \times 10^{7}$ and $4.45 \times 10^{7}$ metagenomic and metatranscriptomic paired-end reads, respectively. In addition, a total of 326,630 additional mass spectra were generated using LC-MS/MS (Methods).

To corroborate the fine-tuning of gene expression of the generalist population CG8b (Candidatus Microthrix parvicella) deduced from the analysis of the sample from SD3, patterns of gene expression were assessed for SD5-SD7. Although the CG5 population consistently expressed the vast majority of its genetic repertoire, only a comparatively small fraction of the genetic complement of CG8b was expressed at each additional time point despite its relatively consistent population size (Table 1, Supplementary Fig. 9b, Supplementary Data 3). These observations support the previous results obtained on the OMMC from SD3. In addition, analogous to the patterns observed for SD3, the 
a
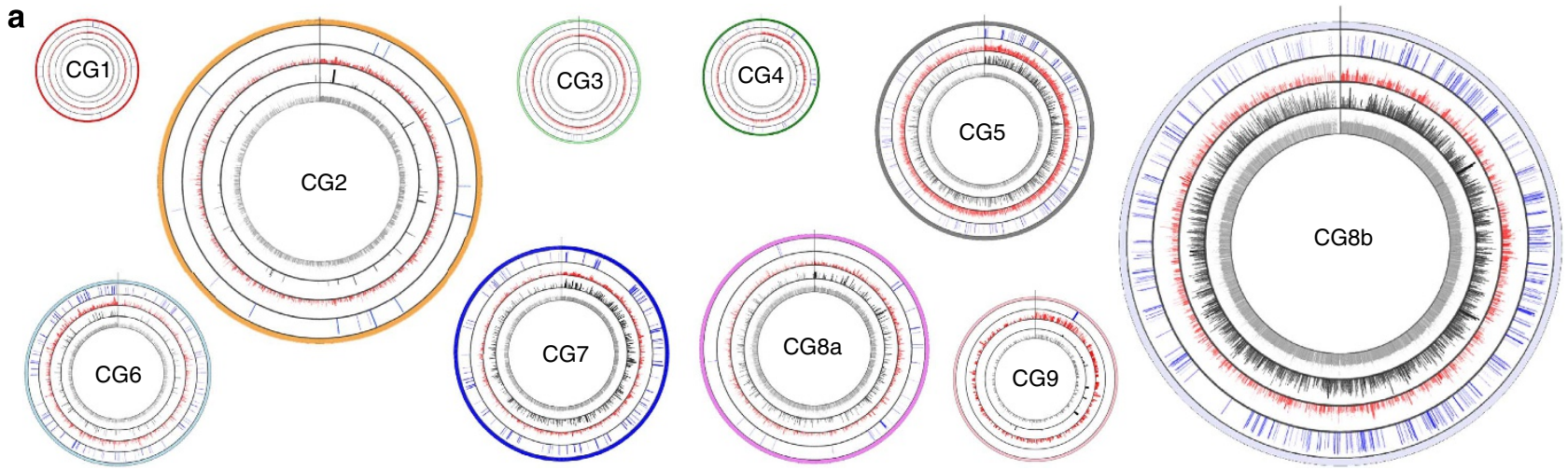

\section{b}

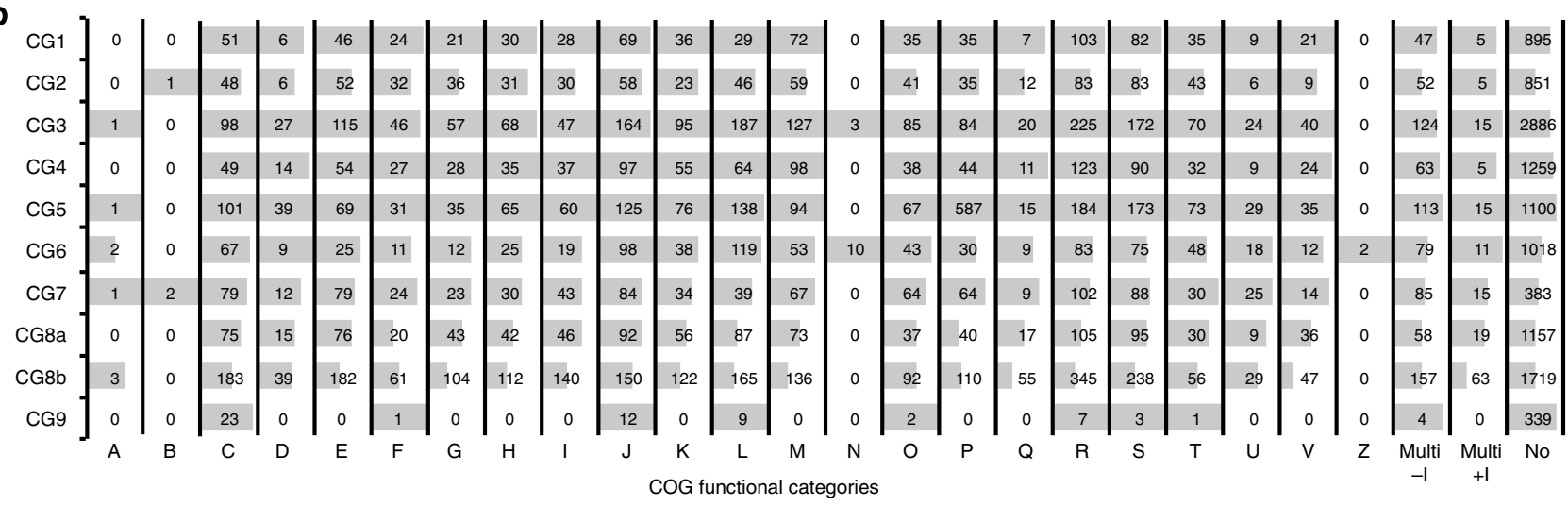

Figure 3 | Population-resolved integrated omics. (a) Circos plots ${ }^{63}$ of genome-wide gene expression levels for the 10 reconstructed composite genomes (CGs). Tracks (from the innermost concentric track to the outermost): $\log _{10}$ of metagenomic fragments per $1 \mathrm{~kb}$ of sequence per $10^{6}$ mapped reads (FPKM ${ }^{46}$; dark grey), $\log _{10}$ of the numbers of detected SNPs per gene (black), $\log _{10}$ of metatranscriptomic FPKM (red), $\log _{2}$ of the protein expression levels as Normalized Spectral Indices (NSI; blue), and reconstructed contigs ordered by size. The track scales are identical across the plots. The sizes of the individual Circos plots are weighted according to the $\log _{10}$ of inferred population size (Methods). (b) The number of genes for each COG category encoded by the different CGs and their corresponding relative transcript levels (grey bars). COG categories are: A, RNA processing and modification; B, chromatin structure and dynamics; $C$, energy production and conversion; D, cell cycle control, cell division, chromosome partitioning; $E$, amino acid transport and metabolism; F, nucleotide transport and metabolism; G, carbohydrate transport and metabolism; $\mathrm{H}$, coenzyme transport and metabolism; I, lipid transport and metabolism; J, translation, ribosomal structure and biogenesis; $\mathrm{K}$, transcription; L, replication, recombination and repair; $\mathrm{M}$, cell wall/membrane/ envelope biogenesis; $\mathrm{N}$, cell motility; $\mathrm{O}$, posttranslational modification, protein turnover, chaperones; $\mathrm{P}$, inorganic ion transport and metabolism; $\mathrm{Q}$, secondary metabolites biosynthesis, transport and catabolism; R, general function prediction only; $\mathrm{S}$, function unknown; $\mathrm{T}$, signal transduction mechanism; $U$, intracellular trafficking, secretion and vesicular transport; $V$, defence mechanisms; $Z$, cytoskeleton; Multi - I, multiple COG category excluding I; Multi + I, multiple COG category including I; No, no COG category assigned.

expression of genes involved in lipid transport and processing encoded by CG8b was highly variable over time (Fig. $4 \mathrm{~b}$ ). In contrast, the CG5 population consistently expressed the vast majority of this functional category (Fig. $4 \mathrm{~b}$, Supplementary Fig. 10). These comprehensive additional data reinforce the notion of finely tuned gene expression for resource usage by the Candidatus Microthrix parvicella generalist population.

The patterns of low SNP density in the generalist population CG8b were also consistent over time, with one order of magnitude fewer SNPs generally apparent in CG8b compared with CG5 (Table 1, Supplementary Data 4). In contrast to CG5, the variant counts of CG8b remain relatively constant over time (Table 1). The observations from the three additional time points reinforce the previous notion that a generalist lifestyle under fluctuating environmental conditions constrains the accumulation of population-level genetic variation.

\section{Discussion}

Here, the application of systematic integrated multi-omic measurements to mixed microbial communities has allowed us to obtain fundamental insights into the ecology of the constituent dominant populations. On the basis of its genetic repertoire and enrichment under temporally changing environmental conditions, the dominant population within the winter OMMCs, that is, Candidatus Microthrix parvicella, can be classified as a generalist species. The low proportion of genes expressed over time indicates that its ecological success most likely results from finely tuned gene expression facilitating optimal foraging behaviour. In addition, the Candidatus Microthrix parvicella population exhibits low levels of genetic variation that may be explained by evolutionary constraints resulting from fitness tradeoffs. Elucidating the exact mechanisms driving these trade-offs in Candidatus Microthrix parvicella, for example, antagonistic pleiotropy or others, will require additional integrated omic data sets to be generated from many more samples taken over space and time, as well as surveys of other wastewater treatment plants. Overall, our results call for similar studies on other microbial communities to determine whether fine-tuning of gene expression is a general feature of generalists and whether lifestyle strategies provide an explanation for the varying degrees of within-population genetic heterogeneity so far observed in metagenomic data sets ${ }^{32}$. 
a

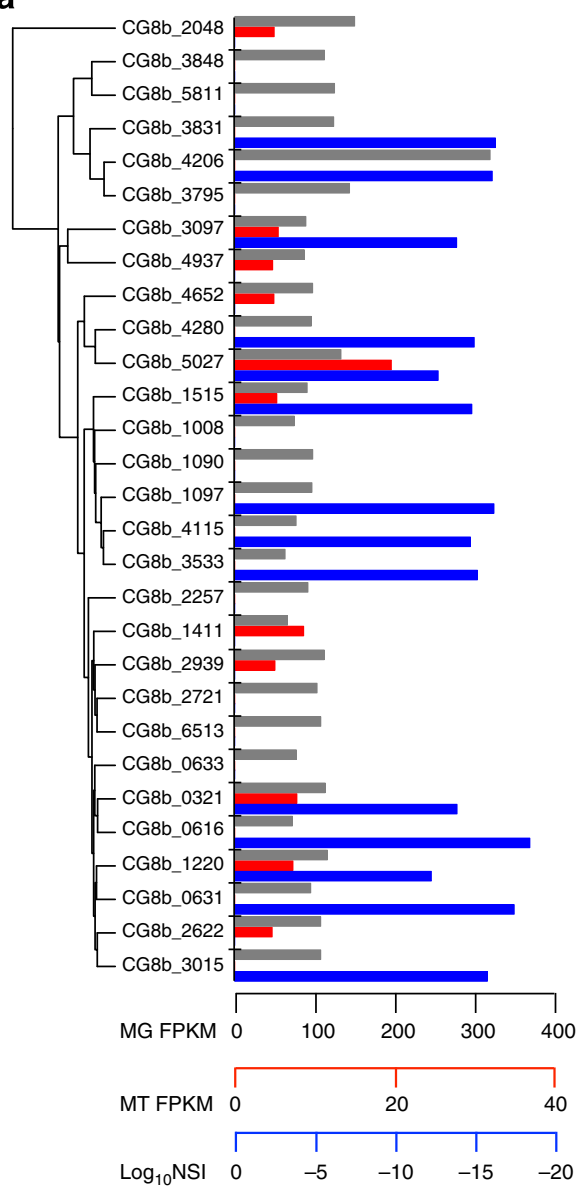

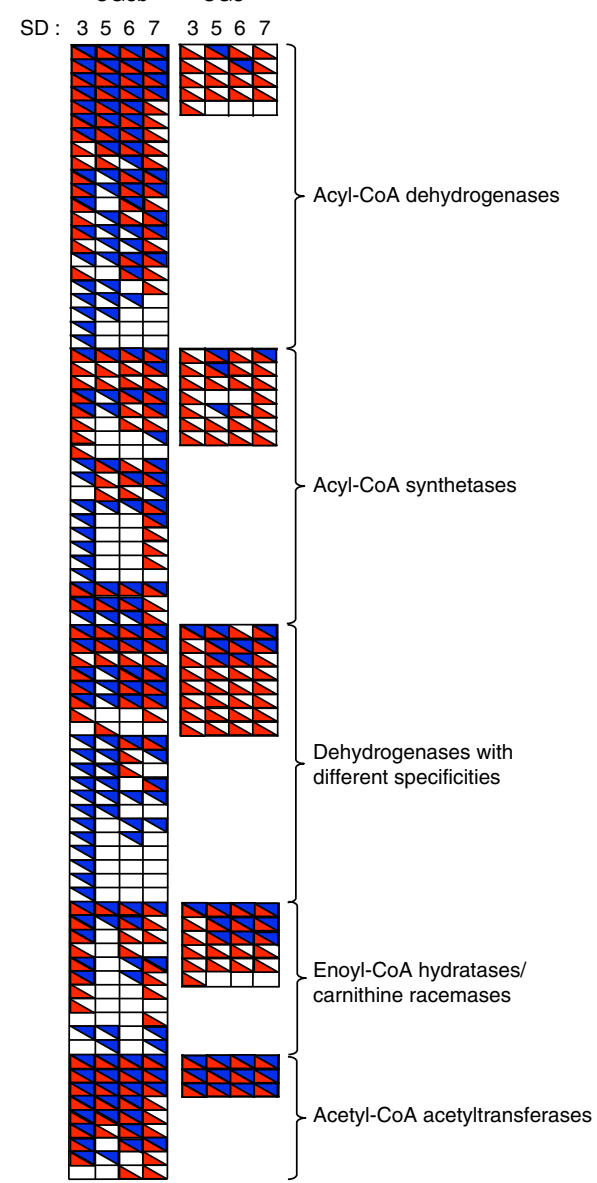

Figure 4 | In situ fine-tuning of gene expression by Candidatus Microthrix parvicella. (a) Dendrogram based on the amino acid similarities of 29 predicted long-chain fatty acid-CoA ligases encoded by the Candidatus Microthrix parvicella population (CG8b). Metagenomic (grey) and metatranscriptomic (red) data represented as fragments per $1 \mathrm{~kb}$ of sequence per $10^{6}$ mapped reads (FPKM), the protein abundance data (blue) is represented as the $\log _{10}$ of the Normalized Spectral Indices (NSI). (b) Qualitative gene expression patterns of the five most prevalent homologous gene sets belonging to the COG category 'I-lipid transport and metabolism' encoded by Candidatus Microthrix parvicella (CG8b) and the CG5 population at the metatranscriptomic (red) and metaproteomic (blue) levels across four sampling time points.

Table 1 | The characteristics of composite genomes CG5 and CG8b at different sampling time points.

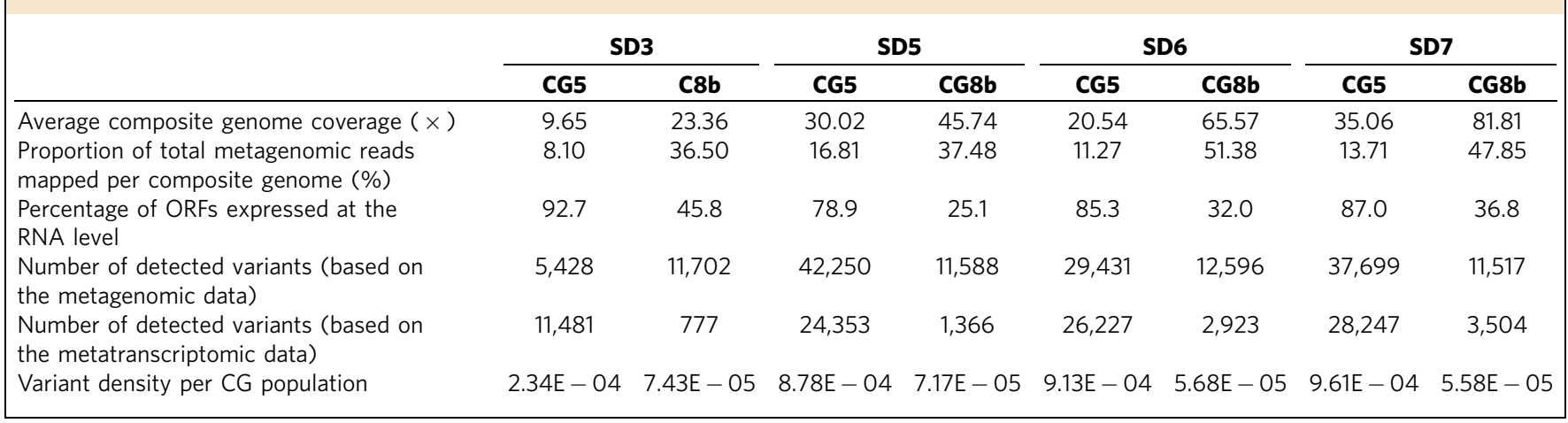

\section{Methods}

Sample processing. Oleaginous mixed microbial communities (OMMCs) were sampled at four representative time points from the surface of the anoxic treatment phase of a biological wastewater treatment plant treating residential effluents (Schifflange, Esch-sur-Alzette, Luxembourg; $49^{\circ} 30^{\prime} 48.29^{\prime \prime} \mathrm{N} ; 6^{\circ} 1^{\prime} 4.53^{\prime \prime} \mathrm{E}$ ).

For each sampling date (SD), four distinct 'islets' were collected (herein referred to as biological replicates; Supplementary Fig. 1), transferred into a sterile tube, snap frozen on site and maintained at $-80^{\circ} \mathrm{C}$ until processing. Initial samples were taken on 4 October 2010 (SD1; anoxic tank wastewater temperature of $20.7^{\circ} \mathrm{C}$ ), 25 October $2010\left(\mathrm{SD} 2 ; 18.9^{\circ} \mathrm{C}\right), 25$ January $2011\left(\mathrm{SD} 3 ; 14.5^{\circ} \mathrm{C}\right)$ and 23 February
2011 (SD4; $\left.13.9^{\circ} \mathrm{C}\right)$. Since the prevalence of OMMCs is dependent on wastewater temperature $^{14}$, these samples were chosen to be representative of the range of wastewater temperatures at which OMMCs are highly abundant within the system. Due to heavy precipitation, which leads to dispersion of the OMMC islets, and due to excess nitrate concentrations (Supplementary Fig. 2a), no samples from December/early January were included in the present study. However, given the range of water temperatures encountered in this biological wastewater treatment plant (Supplementary Fig. 2a), the October as well as January and February samples are representative of autumn and winter OMMCs, respectively. 
For the integrated omic analyses of a representative sample, a single biological replicate (SD3-I; Fig. 1a) from the 25 January 2011 samples was selected for subsequent high-resolution omic analyses. This sample was chosen on the basis of its pronounced community-wide lipid accumulation phenotype, dominance of Candidatus Microthrix spp., and because it exhibited the highest bacterial diversity and evenness. This in turn should allow a comprehensive community-wide overview and reconstruction of composite genomes (CGs) from the most abundant populations within the OMMC.

To validate findings from the integrated omic analysis of the sample from SD3, three additional OMMC samples were rationally selected. On the basis of hierarchical clustering analysis of physico-chemical parameters (Supplementary Fig. 2c), a first additional sample was collected on SD7 (11 January 2012) approximately a year after SD3 when the measured physico-chemical parameters were very similar to those for SD3 (Supplementary Fig. 2b,c). In addition, samples were selected from SD5 (5 October 2011) and SD6 (12 October 2011) because the physico-chemical parameters measured on these dates (especially wastewater temperature) were at variance with those of SD3 and SD7 (Supplementary Fig. $2 b, c)$.

Biomolecular isolation. All biomolecular fractions were obtained using a recently developed methodological framework, which allows recovery of high-quality biomolecular fractions (DNA, RNA, proteins, polar and non-polar metabolites) from unique undivided samples ${ }^{12,13}$. For biomacromolecular purification we used the AllPrep DNA/RNA/Protein Mini kit (Qiagen). Resulting biomolecular fractions comprising genomic DNA, RNA, proteins and small molecules were further processed and analysed as detailed below.

\section{Quantification of biomolecular resources. Intracellular and extracellular non-} polar metabolite fractions of the four biological replicates collected on SD1 to SD4 were obtained using the biomolecular extraction procedure described earlier ${ }^{12,13}$ (only on three biological replicates for SD3). The non-polar phases were aliquoted in four vials (analytical replicates) of $100 \mu$ l each, dried overnight and the resulting pellets were then preserved at $-80^{\circ} \mathrm{C}$. The intracellular and extracellular dried extracts were redissolved in 100 and $40 \mu \mathrm{l}$ of dichloromethane, respectively. Derivatisation was carried out on $40 \mu \mathrm{l}$ of solubilized extract with $40 \mu \mathrm{l}$ of N,O-bis(trimethylsilyl)trifluoroacetamide:trimethylchlorosilane 99:1 (Sylon BFT, Supelco) for $1 \mathrm{~h}$ at $70^{\circ} \mathrm{C}$. Samples were analysed by gas chromatography coupled to tandem mass spectrometry (GC-MS/MS) on a Thermo Trace GC and a Thermo TSQ Quantum XLS triple-quadrupole MS (Thermo Fisher). Samples were injected in PTV splitless mode onto a Rxi-5Sil MS column $(20 \mathrm{~m} \times 0.18 \mathrm{~mm} \times 0.18 \mu \mathrm{m}$, Restek). Helium was used as the carrier gas at a constant flow rate of $1.0 \mathrm{ml} \mathrm{min}^{-1}$. Metabolite detection was performed in Multiple Reaction Monitoring mode, with two Multiple Reaction Monitoring transitions per target compound. Quantification was carried out by external calibration using standard mixtures of pure hexanoic acid, octanoic acid, decanoic acid, dodecanoic acid, tetradecanoic acid, palmitoleic acid, hexadecanoic acid, linoleic acid, oleic acid, linolenic acid, octadecanoic acid, eicosanoic acid, docosanoic acid and tetracosanoic acid, respectively (SigmaAldrich).

Total carbohydrate and protein quantities were determined on supernatant of the same samples comprising $200 \mathrm{mg}$ of OMMC biomass for each sampling date using a Total Carbohydrate Assay Kit and a Total Protein Assay Kit (Micro Lowry, Peterson's Modification; Sigma-Aldrich) according to the manufacturer's instructions.

$16 S$ rRNA amplicon sequencing and analysis. Amplification. The wet-laboratory and bioinformatic procedures for analysing the bacterial community composition based on the V3-V6 region of the bacterial 16S rRNA gene are described in detail elsewhere ${ }^{33}$. Briefly, we generated barcoded V3-V6 amplicons using broadcoverage fusion PCR primers (forward primer: $5^{\prime}$-CCATCTCATCCCTGCGT GTCTCCGACTCAGnnnnnnn $n$ CCTACGGGDGGCWGCA $-3^{\prime}$ and reverse primer 5'- CCTATCCCCTGTGTGCCTTGGCAGTCTCAGCTGACGACRRCCRT GC $\overline{A-3^{\prime}}$ with the underlined portion denoting FLX Lib-L adaptor sequences, italicized portion denoting the sample-specific 8-nt barcode sequences and bold portion denoting $16 \mathrm{~S}$ rRNA gene primer sequences) on $10 \mu \mathrm{l}$ of DNA extracts in $50 \mu \mathrm{l}$ PCR reactions. The barcoded amplicons were pooled and sequenced on a Roche/454 Genome Sequencer FLX platform (Roche Applied Sciences). Resulting pyrosequencing data underwent processing and stringent filtering, which included chimera-checking, demultiplexing and quality-based trimming.

Direct classification. The processed pyrosequences were classified at each taxonomic level using a bootstrap confidence level of $\geq 80$ and using a re-trained version of the Ribosomal Database Project (RDP) Naive Bayesian Classifier 2.4 (refs 34,35), which includes the genus Candidatus Microthrix as a separate taxon. The training set consisted of the RDP $16 \mathrm{~S}$ rRNA training set \#9, with sequences S001942289, S000724117, S000724133, S001448117, S001448118, S001942070, S001942206, S002416756, S002416776, S000014283, S000588187, S000588192, S000010408, S000011228, S000021841, S000267158, S000588182, S000588183, S000588184, S000588185, S000588186, S000588188, S000588189, S000588190, S000588191, S000588193, S000724113, S000724122, S000832952, S000935760, S001294363, S001942173 reclassified as bacteria > Actinobacteria >
Actinobacteria $>$ unclassified $>$ unclassified Candidatus Microthrix, by placing the full 16S rRNA gene sequence of the recently sequenced strain Bio17-1 (ref. 17) into the same taxon. Classification results from each sample were used to produce an abundance matrix for data analysis.

The 16S rRNA gene-based data of the four different biological replicates (islets) per sampling date were used for calculating Simpson diversity and Pielou evenness indices with 10 replications of subsampling of 6,359 reads per sample using the $\mathrm{R}$ Vegan package.

Operational taxonomic unit-based classification. In addition to the direct classification, processed pyrosequences were also analysed by clustering the reads into operational taxonomic units using Mothur ${ }^{36}$ v.1.32.1. To allow appropriate sample-specific classifications, the Candidatus Microthrix parvicella Bio17-1 (ref. 17) $16 \mathrm{~S}$ rRNA gene sequence was added to the Mothur-formatted version of the RDP training set $\mathrm{v} 9$ and the related taxonomy file. Operational taxonomic units clustering was performed at a cut-off level of 0.03 before the assignment of taxonomy. Scripts are available from the authors upon request.

Metagenome and metatranscriptome sequencing and assembly. DNA library preparation. The purified DNA fractions ${ }^{12,13}$ from the unique biological replicates I of SD3 and from SD5 to SD7 suspended in elution buffer ( $\mathrm{pH}$ 8.0) were used to prepare a paired-end library with the AMPure XP/Size Select Buffer Protocol as previously described by Kozarewa et al..$^{37}$, modified to allow for size selection of fragments using the double solid phase reversible immobilization procedure described earlier ${ }^{38}$. Size selection yielded metagenomic library fragments with a mean size of $450 \mathrm{bp}$. All enzymatic steps in the protocol were performed using the Kapa Library Preparation Kit (Kapa Biosystems) with the addition of 1 M PCRgrade betaine in the PCR reaction to aid in the amplification of high G $+\mathrm{C}$ percentage content templates.

RNA library preparation. Following RNA purification ${ }^{12,13}$ from the unique biological replicates I of SD3 and from SD5 to SD7, RNA fractions were ethanol precipitated, overlayed with RNAlater solution (Ambion) and stored at $-80{ }^{\circ} \mathrm{C}$ Before sequencing library preparation, the RNA pellet was rinsed twice in $80 \%$ ethanol and twice in $100 \%$ ethanol to remove any excess RNAlater solution. The pellet was then left on ice to dry. After ethanol evaporation, the RNA pellets were resuspended in $1 \mathrm{mM}$ sodium citrate buffer at $\mathrm{pH}$ 6.4. Ribosomal RNAs were depleted using the Ribo-Zero Meta-Bacteria rRNA Removal Kit (Epicentre) according to the manufacturer's instructions. Transcriptome libraries were subsequently prepared using the ScriptSeq v2

RNA-Seq Library Preparation Kit (Epicentre) according to the manufacturer's instructions. The resulting cDNA was subjected to Illumina sequencing.

Nucleic acid sequencing. Nucleic acid fractions were sequenced on an Illumina Genome Analyser (GA) IIx sequencer. Massive parallel sequencing of DNA and cDNA resulted in the generation of $1.47 \times 10^{7}$ and $1.65 \times 10^{7}$ metagenomic and metatranscriptomic paired-end reads for SD3, respectively. Similarly, the sequencing of SD5-derived DNA and cDNA generated $1.57 \times 10^{7}$ and $1.47 \times 10^{7}$ metagenomic and metatranscriptomic paired-end reads, SD6-derived DNA and cDNA sequencing generated $1.47 \times 10^{7}$ and $1.48 \times 10^{7}$ metagenomic and metatranscriptomic paired-end reads and SD7-derived DNA and CDNA sequencing generated $1.96 \times 10^{7}$ and $1.80 \times 10^{7}$ metagenomic and metatranscriptomic paired-end reads.

Nucleic acid sequence data analysis. MetaPhlAn ${ }^{21}$ (using default parameters) was used on 5' seven base pairs hard-clipped raw paired-end reads, collapsed, filtered at or above a mean QV of 30 and a minimum length of $60 \mathrm{bp}$.

Raw metagenomic paired-end reads were submitted to MG-RAST ${ }^{22}$ using the 'join fastq-formatted paired-reads' option retaining the non-overlapping reads, dynamic trimming and dereplication options. Raw metatranscriptomic reads were submitted to MG-RAST as described for metagenomic data, except that the dereplication option was not selected. As MG-RAST also supports the analysis of eukaryotic sequences, to allow comparison to MetaPhlAn and the 16S rRNA gene sequencing results, the MG-RAST output was filtered to only include bacterial and archaeal taxa. MG-RAST complete functional annotations of both the metagenomic and metatranscriptomic data were used for the assembly-free analysis of the community function.

Apart from these assembly-free community analyses, any overlapping pairedend reads from SD3 were joined with PANDASe ${ }^{39}$ (with threshold parameter $t=0.9$ ) before the removal of potential PCR duplicates using custom scripts (available upon request). Read clipping, quality trimming and filtering of sequence reads was performed with the trim-fastq.pl script from the PoPoolation suite ${ }^{40}$. Four base pairs were hard-clipped from the 5' of all raw reads, and reads were filtered at or above a mean QV of 30 , and a minimum length of $40 \mathrm{bp}$. The quality of the resulting reads and the presence of remaining adaptor sequence contamination were assessed using FastQC (http://www.bioinformatics. babraham.ac.uk/projects/fastqc/).

To reduce the sample complexity and to improve the efficiency of the assembly process, quality-filtered, combined metagenomic and metatranscriptomic reads were initially mapped against the draft genome sequence of Candidatus Microthrix parvicella Bio17-1 (ref. 17). Mapped reads were extracted from the pool of reads and assembled separately with IDBA-UD ${ }^{41}$ (v.1.1.10), with the following parameters: --pre_correction --mink 35 --maxk 75 --step 2 --num_threads 12 -similar 0.97 --min_count 3 . The remaining unmapped reads were binned as pairs 
according to low and high $\mathrm{G}+\mathrm{C}$ percentage content, with an inclusive cut-off value of $50 \% \mathrm{G}+\mathrm{C}$, and assembled as above. This strategy resulted in the generation of 1,739,837 additional base pairs of assembled sequence data compared with a direct assembly. Assemblies were merged using minimus and scaffolded using Bambus2 (AMOS tool suite ${ }^{42}$ ).

Assembled contigs longer than $500 \mathrm{bp}$, representing the first set, were grouped by a reference-free binning algorithm that has recently been developed by some of the authors ${ }^{16}$. The algorithm first computes the pentanucleotide frequency of each contig, which then allows representation of each contig as a point in a 512-dimensional Euclidean space (512 is the number of unique pentanucleotides after taking reverse complements into account). After a centred log-ratio transformation on each point ${ }^{43}$, the sets of points were used as input for the Barnes-Hut Stochastic Neighbourhood Embedding (BH-SNE) algorithm ${ }^{44}$, which produced a two-dimensional map (embedding) of the original signatures (Fig. 2a). Binning of points was then carried out using the Expectation-Maximisation (EM) algorithm on a postulated two-dimensional Gaussian Mixture model ${ }^{45}$, where the means of the Gaussian components of interest were initialized by the user and the covariance matrices were initialized by diagonal matrices with small positive entries. For the results reported in this work, we initialized EM with one Gaussian component per expected cluster following visual inspection. Contigs from the resulting clusters were extracted as contig groups and used as reference sequences to recruit sequence reads from the original, quality-filtered data set. Non-mapped paired-end reads were extracted and merged. A second iterative round of assembly was performed on each set of recruited reads separately and BH-SNE profiling was conducted as described above (except that this time a minimal contig size of $1,000 \mathrm{bp}$ was used). Contig groups resulting from the second BH-SNE iteration were used once more for read recruitment. $\mathrm{G}+\mathrm{C}$ percentage was calculated per base using in-house scripts (available upon request).

For coverage and gene expression analyses (SD3, SD5, SD6 and SD7), metagenomic and metatranscriptomic reads were mapped onto reconstructed genomic fragments from SD3 using Bowtie2 (ref. 46) (using 'very sensitive-local parameters) and $\mathrm{BWA}^{47}$ (using default parameters except for the -M option). Gene expression levels were determined using Cufflinks ${ }^{48}$ on the basis of the BWA read mappings.

Metagenomic FPKM ${ }^{48}$ (fragments per $1 \mathrm{~kb}$ of sequence per $10^{6}$ mapped reads) and coverage values corresponding to each predicted gene in each of the CGs were obtained for the different time points (Supplementary Data 2 and Supplementary Data 3).

To estimate relative population sizes within the community, we devised a measure analogous to the RPKM ${ }^{49}$ (reads per $1 \mathrm{~kb}$ of sequence per $10^{6}$ mapped reads) measure, widely used for reporting the normalized abundance of, for example, transcripts and we defined this as follows:

$$
N_{i}=\frac{c_{i} \times 10^{6}}{C \times l_{i}}
$$

where $N_{i}$ is the relative size of the population corresponding to CGi; $c_{i}$ is the number of reads mapped to $\mathrm{CG} i$; $C$ is the total number of metagenomic reads mappable to all of the CGs; and $l_{i}$ is the length of CGi in bp.

To account for the differences in observed expression levels linked to differing population sizes and to allow comparative analyses between different CGs as well as the different time points, genes were only considered to be expressed per CG per time point, if their metatranscriptomic FPKM values were $\geq 50 \times N_{i}$

(Supplementary Data 2 and Supplementary Data 3).

Metaproteome processing and analysis. Five microlitres of the protein extract obtained from SD3, SD5, SD6 and SD7 as previously described ${ }^{12,13}$ were mixed with $1.25 \mu \mathrm{l}$ of XT sample buffer and $0.25 \mu \mathrm{l}$ of XT reducing agent (Bio-Rad). After $10 \mathrm{~min}$ of denaturation at $70^{\circ} \mathrm{C}, 5 \mu \mathrm{l}$ the sample was subsequently separated by $1 \mathrm{D}$ SDS-PAGE (Criterion precast 1D gel, Bio-Rad). The gel was then stained with Imperial stain (Coomassie-Blue R250, Thermo Fisher Scientific) and cut into uniform $2 \mathrm{~mm}$ bands $\mathrm{s}^{50}$. After in-gel reduction and alkylation, tryptic digestion was performed. Resulting peptides were separated by liquid chromatography (LC) using an Easy-nLC column (Proxeon, Thermo Fisher Scientific). Separation was performed using a $75 \mu \mathrm{m}$ ID fused silica column packed with $20 \mathrm{~cm}$ of ReproSil Pur C18-AQ $3 \mu \mathrm{m}$ beads (Dr Maisch). Before column separation, the samples were loaded onto a fritted $100 \mu \mathrm{m}$ ID fused silica trap packed with $2 \mathrm{~cm}$ of the same material. The peptide mixture was separated using a binary solvent gradient to elute the peptides. Solvent A was $0.1 \%$ formic acid in water. Solvent B was $0.1 \%$ formic acid in acetonitrile. The peptide fractions were pooled in consecutive pairs, concentrated and resuspended up to $20 \mu \mathrm{l}$ in solvent A. Eight microlitres of each pooled sample was injected per LC analysis. The three-step elution programme was operated at a flow rate of $0.3 \mathrm{ml} \mathrm{min}{ }^{-1}$ consisting of (1) a gradient from 2 to $35 \%$ solvent B over $60 \mathrm{~min},(2)$ a $10-\mathrm{min}$ wash at $80 \%$ solvent B and (3) a 20 -min column re-equilibration step at $2 \%$ solvent $B$.

Mass spectra were acquired on an LTQ-Orbitrap Elite (Thermo Fisher Scientific). The instrument was operated on an 11-scan cycle consisting of a single Fourier transformed (FT) precursor scan at 30,000 resolution followed by 10 datadependent MS/MS scan events using higher-energy collisional dissociation at 15,000 resolution in the FT Orbitrap. The precursor scans had a mass range of $300-2,000 \mathrm{~m} / z$, and an automatic gain control setting of $10^{6}$ ions. The MS/MS scans were performed using a normalized collision energy of 35 and an isolation width of $2 \mathrm{~m} / \mathrm{z}$. The data-dependent settings included monoisotopic precursor selection and charge state filtering that excluded unassigned and single charge states. Dynamic exclusion was enabled with a repeat count of 1 , a repeat duration of $10 \mathrm{~s}$, an exclusion list size of 500 and an exclusion duration of $180 \mathrm{~s}$. Exclusion mass width was \pm 5 p.p.m. relative to mass.

LC-MS/MS analysis resulted in the generation of 271,915 mass spectra for SD3, 118,386 mass spectra for SD5, 102,916 mass spectra for SD6 and 105,328 mass spectra for SD7.

Composite genome and expression analyses. Gene calling and annotation. The assembled CGs were submitted for gene calling and annotation to RAST ${ }^{51}$ with default parameters except for Domain (Bacteria), Genetic code (11), Sequencing method (other), FIGfam version (release 63) and with the Build metabolic model option selected.

Taxonomic affiliation. The taxonomies of the reconstructed CGs were determined using the AmphoraNet ${ }^{24}$ webserver. A taxon name was assigned when at least $75 \%$ of the identified marker genes resulted in a concordant taxonomy, and a putative taxon name was assigned when at least $50 \%$ of the identified markers resulted in a concordant taxonomy.

Completeness and composition of composite genomes. Genome completeness of the reconstructed CGs was estimated on the basis of 40 universal single copy genes $^{52}$. For this, the functional annotation of the predicted proteins in each CG was obtained using the WebMGA server ${ }^{53}$ using the 'cog' analysis option. Functional compositions of the CGs and of their expressed genes were obtained from COG category counts, which were normalized by the total number of predicted features per CG.

Comparative analysis of Candidatus Microthrix parvicella-like sequences. Draft genome sequences for Candidatus Microthrix parvicella strains Biol7-1 (ref. 17) and RN1 (ref. 18) were obtained from the GenBank database (Assembly ID GCA_000299415.1 and GCA_000455525.1, respectively). Sets of orthologous genes were built using RAST's 'sequence based comparison' tool.

Variant identification. SNPs were identified by separately mapping metagenomic and metatranscriptomic reads against the reconstructed CG assemblies using Bowtie2 and BWA (as described above). SNPs were identified from each of the mappings using mpileup (SAMtools ${ }^{54}$ ), the UnifiedGenotyper (Genome Analysis Tool Kit ${ }^{55}$ ) and Freebayes ${ }^{56}$. The intersection of identified SNPs from all the aforementioned methods was obtained using the vcf-isec utility from the VCFtools suite and was considered for subsequent analyses. SD3 variant amino-acid sequences were included in the amino-acid sequence databases generated on the basis of called and annotated genes. This database was used for subsequent protein identification on the basis of the generated metaproteomic data (see below). Variant frequencies were separately estimated from mapped metagenomic and metatranscriptomic reads. Only variants in regions with a minimum read depth (coverage) of 10 for both metagenomic and metatranscriptomic data were considered. Variant density per CG population was calculated by normalizing the SNP density (number of SNPs per kb) by the relative population size, which in turn was inferred from the fraction of metagenomic sequencing reads mapped onto the individual genomic reconstructions.

Protein identification. MS/MS spectra were searched against the generated amino-acid sequence database (containing the predicted proteins including all variants of the 10 reconstructed CGs and common contaminants) using the $\mathrm{X}$ !Tandem algorithm ${ }^{57}$. The resulting peptide identifications were validated using the Trans-Proteomic Pipeline ${ }^{58}$. The X!Tandem parameters included precursor and fragment ion mass tolerances of 15 p.p.m., a static modification of $57.021464 \mathrm{Da}$ on cysteine residues and a potential modification mass of 15.994915 $\mathrm{Da}$ on methionine residues. The search allowed for semi-tryptic cleavages up to two missed cleavages. The database search results were validated and proteins were inferred at $\sim 1 \%$ false discovery rate using the PeptideProphet, ProteinProphet and iProphet tools from the Trans-Proteomic Pipeline software suite $^{58-60}$.

Protein quantification. Relative protein quantification was performed using the normalized spectral index (NSI) measure using an in-house software tool called NSICalc (details available upon request). The tool was adapted from the method by Griffin et al ${ }^{61}$ Briefly, the NSI combines peptide count, spectral count and MS/MS fragment ion intensity for quantification and normalizes these values by the length of each protein. This strategy incorporates measurable peptide intensities while removing some of the biases of using spectral counts when comparing large and small proteins. NSI values were $\log _{2}$ normalized before comparison across proteins to obtain relative quantification ratios.

Metaproteomic analyses led to peptide matching against the amino-acid database of 43,214 spectra, which in turn provided abundance data on a total of 1,815 proteins for SD3.

Analysis of the long-chain fatty acid-CoA ligases of CG8b. Amino-acid sequences of genes annotated as long-chain-fatty-acid-CoA ligases by RAST from CG8b were aligned using Expresso ${ }^{62}$ using default parameters. Sequence similarities were determined using the SIAS server (http://imed.med.ucm.es/Tools/sias.html). A dendrogram based on pairwise comparisons of amino-acid sequence similarities was obtained using the hierarchical clustering function in R. Abundance values were extracted from the mapped metagenomic, metatranscriptomic and metaproteomic data. 


\section{References}

1. Muller, E. E. L., Glaab, E., May, P., Vlassis, N. \& Wilmes, P. Condensing the omics fog of microbial communities. Trends Microbiol. 21, 325-333 (2013).

2. Falkowski, P. G., Fenchel, T. \& Delong, E. F. The microbial engines that drive Earth's biogeochemical cycles. Science 320, 1034-1039 (2008).

3. Nicholson, J. K., Holmes, E. \& Wilson, I. D. Gut microorganisms, mammalian metabolism and personalized health care. Nat. Rev. Microbiol. 3, 431-438 (2005).

4. Rittmann, B. E. Microbial ecology to manage processes in environmental biotechnology. Trends Biotechnol. 24, 261-266 (2006).

5. Waldrop, M. P., Zak, D. R., Blackwood, C. B., Curtis, C. D. \& Tilman, D. Resource availability controls fungal diversity across a plant diversity gradient. Ecol. Lett. 9, 1127-1135 (2006).

6. Langenheder, S. \& Prosser, J. I. Resource availability influences the diversity of a functional group of heterotrophic soil bacteria. Environ. Microbiol. 10, 2245-2256 (2008).

7. Rasche, F. et al. Seasonality and resource availability control bacterial and archaeal communities in soils of a temperate beech forest. ISME J. 5, 389-402 (2011)

8. Dumbrell, A. J., Nelson, M., Helgason, T., Dytham, C. \& Fitter, A. H. Relative roles of niche and neutral processes in structuring a soil microbial community. ISME J. 4, 1078-1078 (2010).

9. Jeraldo, P. et al. Quantification of the relative roles of niche and neutral processes in structuring gastrointestinal microbiomes. Proc. Natl Acad. Sci. USA 109, 9692-9698 (2012).

10. Mou, X., Sun, S., Edwards, R. A., Hodson, R. E. \& Moran, M. A. Bacterial carbon processing by generalist species in the coastal ocean. Nature 451, 708-711 (2008)

11. Wilmes, P. et al. Metabolome-proteome differentiation coupled to microbial divergence. MBio 1, e00246-10 (2010).

12. Roume, H. et al. A biomolecular isolation framework for eco-systems biology. ISME J. 7, 110-121 (2013).

13. Roume, H., Heintz-Buschart, A., Muller, E. E. L. \& Wilmes, P. Sequential isolation of metabolites, RNA, DNA, and proteins from the same unique sample. Methods Enzymol. 531, 219-236 (2013).

14. Rossetti, S., Tomei, M. C., Nielsen, P. H. \& Tandoi, V. 'Microthrix parvicella', a filamentous bacterium causing bulking and foaming in activated sludge systems: a review of current knowledge. FEMS Microbiol. Rev. 29, 49-64 (2005).

15. Muller, E. E. L., Sheik, A. R. \& Wilmes, P. Lipid-based biofuel production from wastewater. Curr. Opin. Biotechnol. 30, 9-16 (2014)

16. Laczny, C. C., Pinel, N., Vlassis, N. \& Wilmes, P. Alignment-free visualization of metagenomic data by nonlinear dimension reduction. Sci. Rep. 4, 4516 (2014)

17. Muller, E. E. L. et al. Genome sequence of 'Candidatus Microthrix parvicella' Bio17-1, a long-chain-fatty-acid-accumulating filamentous actinobacterium from a biological wastewater treatment plant. J. Bacteriol. 194, 6670-6671 (2012).

18. Jon McIlroy, S. et al. Metabolic model for the filamentous 'Candidatus Microthrix parvicella' based on genomic and metagenomic analyses. ISME J. 7, 1161-1172 (2013).

19. Emlen, J. M. The role of time and energy in food preference. Am. Nat. 100, 611 (1966).

20. MacArthur, R. H. \& Pianka, E. R. On optimal use of a patchy environment. Am. Nat. 100, 603-609 (1966).

21. Segata, N. et al. Metagenomic microbial community profiling using unique clade-specific marker genes. Nat. Methods 9, 811-814 (2012).

22. Meyer, F. et al. The metagenomics RAST server - a public resource for the automatic phylogenetic and functional analysis of metagenomes. $B M C$ Bioinformatics 9, 386 (2008).

23. Konstantinidis, K. T. \& Tiedje, J. M. Towards a genome-based taxonomy for prokaryotes. J. Bacteriol. 187, 6258-6264 (2005).

24. Kerepesi, C., Bánky, D. \& Grolmusz, V. AmphoraNet: the webserver implementation of the AMPHORA2 metagenomic workflow suite. Gene $\mathbf{5 3 3}$ 538-540 (2014)

25. Pradet-Balade, B., Boulmé, F., Beug, H., Müllner, E. W. \& Garcia-Sanz, J. A. Translation control: bridging the gap between genomics and proteomics? Trends Biochem. Sci. 26, 225-229 (2001).

26. Urich, T. et al. Microbial community structure and functioning in marine sediments associated with diffuse hydrothermal venting assessed by integrated meta-omics. Environ. Microbiol. 16, 2699-2710 (2013).

27. Kassen, R. The experimental evolution of specialists, generalists, and the maintenance of diversity. J. Evol. Biol. 15, 173-190 (2002).

28. Black, P. N. \& DiRusso, C. C. Transmembrane movement of exogenous longchain fatty acids: proteins, enzymes, and vectorial esterification. Microbiol. Mol. Biol. Rev. 67, 454-472 (2003).

29. Whitlock, M. C. The red queen beats the jack-of-all-trades: the limitations on the evolution of phenotypic plasticity and niche breadth. Am. Nat. 148 S65-S77 (1996).
30. Elena, S. F. \& Lenski, R. E. Evolution experiments with microorganisms: the dynamics and genetic bases of adaptation. Nat. Rev. Genet. 4, 457-469 (2003).

31. Fraser, C., Alm, E. J., Polz, M. F., Spratt, B. G. \& Hanage, W. P. The bacterial species challenge: making sense of genetic and ecological diversity. Science $\mathbf{3 2 3}$ 741-746 (2009)

32. Wilmes, P., Simmons, S. L., Denef, V. J. \& Banfield, J. F. The dynamic genetic repertoire of microbial communities. FEMS Microbiol. Rev. 33, 109-132 (2009).

33. Liu, C. M. et al. Male circumcision significantly reduces prevalence and load of genital anaerobic bacteria. MBio 4, e00076 (2013).

34. Cole, J. R. et al. The Ribosomal Database Project: improved alignments and new tools for rRNA analysis. Nucleic Acids Res. 37, D141-D145 (2009).

35. Wang, Q., Garrity, G. M., Tiedje, J. M. \& Cole, J. R. Naive Bayesian classifier for rapid assignment of rRNA sequences into the new bacterial taxonomy. Appl. Environ. Microbiol. 73, 5261-5267 (2007).

36. Schloss, P. D. et al. Introducing mothur: open-source, platform-independent, community-supported software for describing and comparing microbial communities. Appl. Environ. Microbiol. 75, 7537-7541 (2009).

37. Kozarewa, I. et al. Amplification-free Illumina sequencing-library preparation facilitates improved mapping and assembly of GC-biased genomes. Nat. Methods 6, 291-295 (2009).

38. Rodrigue, S. et al. Unlocking short read sequencing for metagenomics. PLoS ONE 5, el1840 (2010).

39. Masella, A. P., Bartram, A. K., Truszkowski, J. M., Brown, D. G. \& Neufeld, J. D. PANDAseq: paired-end assembler for illumina sequences. BMC Bioinformatics 13, 31 (2012).

40. Kofler, R. et al. PoPoolation: a toolbox for population genetic analysis of next generation sequencing data from pooled individuals. PLoS ONE 6, e15925 (2011).

41. Peng, Y., Leung, H. C. M., Yiu, S. M. \& Chin, F. Y. L. IDBA-UD: a de novo assembler for single-cell and metagenomic sequencing data with highly uneven depth. Bioinformatics 28, 1420-1428 (2012).

42. Treangen, T. J., Sommer, D. D., Angly, F. E., Koren, S. \& Pop, M. Next generation sequence assembly with AMOS. Curr. Protoc. Bioinformatics Chapter 11, Unit 11.8 (2011)

43. Aitchison, J. The Statistical Analysis of Compositional Data (Blackburn Press, 2003).

44. Van der Maaten, L. Barnes-Hut-SNE. in Proceedings of the International Conference on Learning Representations arXiv:1301.3342 (2013).

45. Redner, R. A. \& Walker, H. F. Mixture densities, maximum likelihood and the EM algorithm. SIAM Rev. 26, 195-239 (1984).

46. Langmead, B. \& Salzberg, S. L. Fast gapped-read alignment with Bowtie 2. Nat Methods 9, 357-359 (2012).

47. Li, H. \& Durbin, R. Fast and accurate long-read alignment with BurrowsWheeler transform. Bioinformatics 26, 589-595 (2010).

48. Trapnell, C. et al. Transcript assembly and quantification by RNA-Seq reveals unannotated transcripts and isoform switching during cell differentiation. Nat. Biotechnol. 28, 511-515 (2010).

49. Mortazavi, A., Williams, B. A., McCue, K., Schaeffer, L. \& Wold, B. Mapping and quantifying mammalian transcriptomes by RNA-Seq. Nat. Methods 5, 621-628 (2008)

50. Simpson, R. J. et al. Proteomic analysis of the human colon carcinoma cell line (LIM 1215): development of a membrane protein database. Electrophoresis 21 1707-1732 (2000).

51. Aziz, R. K. et al. The RAST Server: rapid annotations using subsystems technology. BMC Genomics 9, 75 (2008).

52. Stark, M., Berger, S. A., Stamatakis, A. \& von Mering, C. MLTreeMapaccurate Maximum Likelihood placement of environmental DNA sequences into taxonomic and functional reference phylogenies. BMC Genomics 11, 461 (2010).

53. Wu, S., Zhu, Z., Fu, L., Niu, B. \& Li, W. WebMGA: a customizable web server for fast metagenomic sequence analysis. BMC Genomics 12, 444 (2011).

54. Li, H. et al. The sequence alignment/map format and SAMtools. Bioinformatics 25, 2078-2079 (2009).

55. McKenna, A. et al. The Genome Analysis Toolkit: a MapReduce framework for analyzing next-generation DNA sequencing data. Genome Res. 20, 1297-1303 (2010).

56. Garrison, E. \& Marth, G. Haplotype-based variant detection from short-read sequencing. ArXiv e-print 1207:3907 (2012).

57. Craig, R. \& Beavis, R. C. A method for reducing the time required to match protein sequences with tandem mass spectra. Rapid Commun. Mass Spectrom 17, 2310-2316 (2003).

58. Nesvizhskii, A. I., Keller, A., Kolker, E. \& Aebersold, R. A statistical model for identifying proteins by tandem mass spectrometry. Anal. Chem. 75, 4646-4658 (2003).

59. Keller, A., Nesvizhskii, A. I., Kolker, E. \& Aebersold, R. Empirical statistical model to estimate the accuracy of peptide identifications made by MS/MS and database search. Anal. Chem. 74, 5383-5392 (2002). 
60. Shteynberg, D. et al. iProphet: multi-level integrative analysis of shotgun proteomic data improves peptide and protein identification rates and error estimates. Mol. Cell. Proteomics 10, M111.007690 (2011).

61. Griffin, N. M. et al. Label-free, normalized quantification of complex mass spectrometry data for proteomic analysis. Nat. Biotechnol. 28, 83-89 (2010).

62. Armougom, F. et al. Expresso: automatic incorporation of structural information in multiple sequence alignments using 3D-Coffee. Nucleic Acids Res. 34, W604-W608 (2006).

63. Lin, J. et al. POMO-plotting omics analysis results for multiple organisms. BMC Genomics 14, 918 (2013).

\section{Acknowledgements}

We thank the scientists and technical staff of the Luxembourg Centre for Systems Biomedicine and TGen North, particularly Dr René Hussong and Dr Owain Moss for their assistance and support; Mr Bissen and Mr Di Pentima from the Syndicat Intercommunal à Vocation Ecologique (SIVEC), for their permission to collect samples and access to the monitoring platform of the Schifflange wastewater treatment plant; Joëlle Fritz, Sheri Simmons and Rudi Balling for their comments on the manuscript. Bioinformatic analyses presented in this paper were carried out using the highperformance computing facilities of the University of the Luxembourg. This work was supported by an ATTRACT programme grant (ATTRACT/A09/03) and a European Union Joint Programming in Neurodegenerative Diseases grant (INTER/JPND/12/01) both to P.W., and Aide à la Formation Recherche (AFR) grants to H.R. (PHD/MARP04), C.C.L. (PHD/4964712), S.N. (PHD-2014-1/7934898), L.W. (PHD-2013-1/BM) and E.E.L.M. (PRD-2011-1/SR), all funded by the Luxembourg National Research Fund (FNR). The project received financial support from the Integrated Biobank of Luxembourg with funds from the Luxembourg Ministry of Higher Education and Research. The work of N.P., J.L. and P.M. was funded by the 'Plan Technologies de la Santé du Gouvernement du Grand-Duché de Luxembourg' through the Luxembourg Centre for Systems Biomedicine (LCSB), University of Luxembourg. The work was also, in part, supported by US federal funds from the National Science Foundation (MRI grant No. 0923536), by funds from the National Institutes of Health and by funds from the US National Institute of General Medical Sciences (grant No. 2P50 GM076547/Center for Systems Biology and S10RR027584) to R.L.M. and M.R.H.

\section{Author contributions}

E.E.L.M., H.R., L.A.L. and P.W. sampled the treatment plant and extracted biomolecules; E.E.L.M., N.P., C.C.L., S.N., J.L., P.M., N.D.H., A.H.-B., L.W., C.M.L., N.V. and P.W. analysed the (meta)genomic and/or metatranscriptomic data; E.E.L.M., N.P., M.R.H. and R.L.M. analysed the metaproteomic data; E.E.L.M., N.P. and C.G. analysed the meta bolomic data; E.E.L.M, N.P. and P.W. designed the study and wrote the manuscript. All the authors discussed the results and commented on the manuscript.

\section{Additional information}

Accession codes: All raw sequence data have been deposited in the NCBI Bioproject with the accession code PRJNA230567. MG-RAST-based analysis results of the metagenomic and metatranscriptomic data can be found at http://metagenomics.anl.gov/linkin.cgi?metagenome $=4566023.3$ and http://metagenomics.anl.gov/linkin.cgi?metagenome $=4566620.3$, respectively. RAST IDs from 6666666.37195 to 6666666.37203 correspond to CG1 to CG9 assemblies and annotations are available through the RAST guest account at http://rast.nmpdr.org, using 'guest' as login as well as password. All proteomic data has been deposited in the PeptideAtlas mass spectrometry raw file repository at http://www.peptideatlas.org/ under the 'datasetIdentifier' PASS00359, PASS00576, PASS00577 and PASS00578 for SD3, SD5, SD6 and SD7, respectively.

Supplementary Information accompanies this paper at http://www.nature.com/ naturecommunications

Competing financial interests: The authors declare no competing financial interests.

Reprints and permission information is available online at http://npg.nature.com/ reprintsandpermissions/

How to cite this article: Muller, E. E. L. et al. Community-integrated omics links dominance of a microbial generalist to fine-tuned resource usage. Nat. Commun. 5:5603 doi: $10.1038 /$ ncomms6603 (2014).

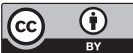

This work is licensed under a Creative Commons Attribution 4.0 International License. The images or other third party material in this article are included in the article's Creative Commons license, unless indicated otherwise in the credit line; if the material is not included under the Creative Commons license, users will need to obtain permission from the license holder to reproduce the material. To view a copy of this license, visit http://creativecommons.org/licenses/by/4.0/ 